\title{
Effect of signal delay on auditory detection with gated noise
}

\author{
W. A. WILBANKS \\ UNIVERSITY OF MISSISSIPPI
}

Monaural and binaural detection of a gated signal is more difficult when the noise is gated simultaneously with the signal than when the noise is continuous. As the onset of the signal is delayed, binaural detection improves quite rapidly. Monaural detection improves with signal delay, but at $a$ slower rate. The results are interpreted in terms of signal detection theory.

Several investigators have reported that gated noise may produce more masking of tonal signals than continuous noise at the same spectral level. The greater effectiveness of gated noise has been shown to depend upon the interaural condition of signal and noise presentation, and upon the temporal relation between the onset of signal and noise. Sherrick \& Albernaz (1961) found that if a noise is presented to one ear and the signal to the other (so-called "central" masking), about 6dB more masking occurs if the nolse and signal are gated simultaneously than if the noise is presented continuously and only the signal is gated. Watson (1962) reported about 2-4 dB more masking with gated than with continuous noise when both signal and noise are presented in-phase at both ears (NO-SO). MCFadden (1966), on the other hand, found little difference between continuous and gated noise for NO-SO. McFadden reports, however, that if either the signal or the noise (but not both) is reversed in phase by 180 degrees, a gated noise is about 5-6 dB more effective than a continuous noise. Zwislockd (1965) and McFadden (1966) have shown that if the noise and the signal are not gated simultaneously, i.e., if the onset of the signal is delayed re the onset of the noise, the effect of noise gating diminishes to the point where there is virtually no difference between detection with continuous and gated noise. The present study extends the work on the effect of signal delay on detection with gated noise to the case where both signal and noise are presented monaurally (NM-SM), and where the noise is presented binaurally in-phase and the signal to only one ear (NO-SM).

Wethod

The signal, a 250-Hz pure tone, was presented monaurally at the listener's more sensitive ear. The duration of the signal (50 msec) was controlled by an Iconix electronic counter that triggered a GrasonStadler switch, gating the signal with a $10 \mathrm{msec}$ risefall time.

The signal was partially masked by wide-band nolse, cut-off at the lower end by the response of the earphones (TDH-49), and at the upper end by a 3000Hz low-pass passive filter. The spectral level of the masker, measured at 250-Hz across the earphones with a Hewlett-Packard wave-analyzer, corresponded to approximately $+45 \mathrm{~dB}$ re $\mathbf{0 . 0 0 0 2}$ microbar per cycle. The noise was presented monaurally at the signal ear, and binaurally in-phase at both ears. This latter condition provides a noise whose product-moment correlation is $+1.00(\mathrm{NO})$. Both continuous and gated maskers were used. The duration of the gated noise (200 msec) was controlled by the same electronic counter that controlled the duration of the signal. The counter triggered a Grason-Stadler switch that gated the noise with a $10 \mathrm{msec}$ rise-fall time. For the gated noise condition, the onset of the signal was delayed re the onset of the noise by $0,25,50$, $75,100,125$, and $150 \mathrm{msec}$.

The detectability of the stgnal was determined by means of a two-alternative, temporal forced-choice (2AFC) method. The signal was programmed to occur at random in one or the other of two temporal intervals marked for the listener by lights. With the gated noise, the observation interval lights always coincided with the time of signal onset and offset, and not with the onset-offset of the noise. This was done, of course, to reduce the uncertainty of the listeners as to the time of aignal presentation (Egan, Greenberg, \& Schulman, 1961). The listeners were informed that the a priori probability of the signal occurring during the first interval was 0.50 .

Three well-trained listeners were used, two men and one woman. Since they were experienced with 2AFC experiments, no special instructions were required, and no system of trial-by-trial informational feedback was employed.

For each experimental condition, five signal levels, separated by equal dB steps, were used. Psychometric functions were then obtained relating percentage correct choices to signal level $\left(E / N_{O}\right)$. The correction for response bias suggested by Egan (1965) was used. From these psychometric functions, the main dependent variable was determined: the signal level in $\mathrm{dB}$ required for $85 \%$ correct choices.

\section{Results and Discussion}

The results are presented in Figs. 1 and 2. In Fig. 1, the ordinate is the signal level in $\mathrm{dB}$ for $85 \%$ correct decisions, the abscissa is the delay $(T)$ in msec between the onset of the signal and of the noise, and the parameter is the interaural condition of the noise, 1.e., noise monaural (NM) or noise 


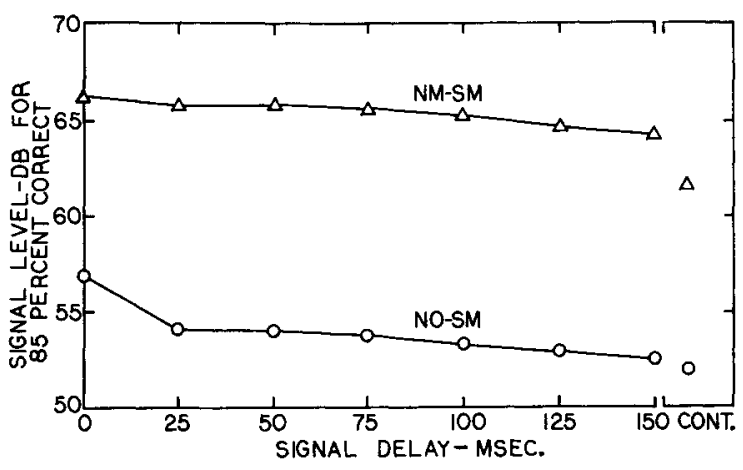

Fig. 1. Signal level in dB for $85 \%$ correct detections as a function of signal delay $(T)$ in msec and interaural condition. NM $-\mathrm{SM}=$ noise monaural, signal monaural; NO-SM=noise correlated, signal monaural.

binaural in-phase (NO). The two points at the extreme right are the results with continuous monaural and correlated noise. In Fig. 2, the ordinate is the amount of masking in $\mathrm{dB}$ produced by gated noise re that produced by continuous noise, the abscissa is the time delay $(\tau)$ in msec between signal and noise onset, and the parameter is again NM and NO.

With a monaural masker, gating the noise so that its onset is synchronous with that of the signal ( $T=0$ msec) reduces the detectability of the signal by about $4 \mathrm{~dB}$. Simultaneous gating of signal and correlated noise degrades detection by about $5 \mathrm{~dB}$. With NO-SM, there is a rapid improvement in detection as the onset of the signal is delayed. For $T=150 \mathrm{msec}$, the difference between gated and continuous noise is less than $1 \mathrm{~dB}$. With a gated monaural noise, detection improves with signal delay, but at a slower rate. The decay in the effectiveness of a gated masker with signal delay provides a simple explanation for the difference in the detectability of signals partially masked by steady and gated maskers: a gated noise is more effective than a continuous noise when its onset is synchronous with that of the signal.

The difference between detection with NO-SM and NM-SM as a function of signal delay is interesting. With continuous noise, detection is about $10 \mathrm{~dB}$ better with NO than with NM. This masking-level difference (MLD) agrees very closely with that reported by Whitmore \& Wilbanks (1965a, b), Wilbanks \& Whitmore (1967), Blodgett, Jeffress, \& Whitmore (1962), and by other investigators. As the signal is delayed, the MLD with gated noise increases to about $12 \mathrm{~dB}$. This increase is consistent with McFadden's (1966) findings.

According to detection theory, the basis for detection is some sort of transformation of the noise distribution when the signal is added. In the approach taken by Peterson, Birdsall, \& Fox (1954), and by Jeffress (1964), detection is based upon some difference between a sample of noise (N) and signal-

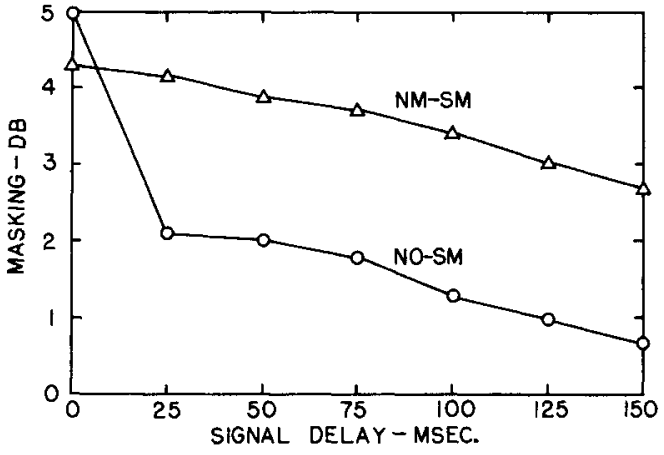

Fig. 2. Masking in $\mathrm{dB}$ by gated noise re continuous noise as a function of signal delay $(T)$ in msec and interaural condition. $N M \rightarrow S M=$ noise monaural, signal monaural; NO-SM=noise correlated, signal monaural.

plus-noise $(S+N)$. For monaural detection, the crucial difference between $\mathrm{N}$ and $\mathrm{S}+\mathrm{N}$ appears to be one of amplitude. In the case of binaural detection (NO-SM), the difference is between the running cross-correlations of $\mathrm{N}$ and $\mathrm{S}+\mathrm{N}$. The results of the present study show that noise gating degrades detection when the change in $\mathrm{N}$ due to the onset of the signal $(\mathrm{S}+\mathrm{N})$ is minimal, i.e., when the signal and noise are gated simultaneously.

\section{References}

Blodgett, H. C., Jeffress, L. A., \& Whitworth, R. H. Effect of noise at one ear on the masked threshold for tone at the other. J. Acoust. Soc. Amer., 1962, 34, 979-981.

Egan, J. P. Masking-level differences as a function of interaural disparities in intensity of signal and noise. J. Acoust. Soc. Amer., 1965, 38, 1043-1049.

Egan, J. P., Greenberg, G. Z., \& Schulman, A. I. Interval of time uncertainty in auditory detection. J. Acoust. Soc. Amer., 1961, 33, 771-778.

Jeffress, L. A. Stimulus-oriented approach to detection. J. Acoust. Soc. Amer., 1964, 36, 766-774.

McFadden, D. Masking-level differences with continuous and with burst masking noise. J. Acoust. Soc. Amer., 1966, 40, 1414-1419.

Peterson, W. A., Birdsall, T. G., \& Fox, W. C. The theory of signal detectability. Trans. IRE, 1954, PGIT-4, 171-212.

Sherrick, C. E., \& Mangabeira-Albemaz, P. L. Auditory threshold shifts produced by simultaneously pulsed contralateral stimuli. J. Acoust. Soc. Amer., 1961, 33, 1381-1385.

Watson, C. S. Signal detection and certain physical characteristics of the stimulus during the observation interval. Ph. D. dissertation, Indiana University, 1962.

Whitmore, J. K., \& Wilbanks, W. A. Interaural noise correlation and monaural signal detection. J. Acoust. Soc. Amer., 1965a, 37, $1179($ A).

Whitmore, J. K., \& Wilbanks, W. A. Interaural noise correlation and detection of low-frequency monaural signals. J. Acoust. Soc. Amer., 1965b, 38, 929(A).

Wilbanks, W. A., \& Whitmore, J. K. Detection of monaural signals as a function of interaural noise-correlation and signal frequency. J. Acoust. Soc. Amer., 1967, in press.

Zwislocki, J. Time and frequency parameters of central masking. Paper read at Psychon. Soc., Oct. 1965; also personal communication. 